\title{
Proteome-Based Pathway Modelling of Psychiatric Disorders
}

Authors

Affiliations
C. W. Turck ${ }^{1}$, F. Iris ${ }^{2}$

${ }^{1}$ Max Planck Institute of Psychiatry, Proteomics and Biomarkers, Munich, Germany ${ }^{2}$ Bio-Modeling Systems, Paris, France
Bibliography

DOI http://dx.doi.org/

10.1055/s-0031-1271701

Pharmacopsychiatry 2011;

44 (Suppl. 1): S1-S8

(c) Georg Thieme Verlag KG Stuttgart · New York

ISSN 0176-3679

Correspondence

Dr. C. W. Turck

Proteomics and Biomarkers

Max Planck Institute of

Psychiatry

Kraepelinstraße 2-10

80804 Munich

Germany

Tel.: +49/89/306 22317

Fax:+49/89/306 22610

turck@mpipsykl.mpg.de

\section{Abstract \\ $\nabla$}

The etiopathogenesis of many psychiatric illnesses remains unclear and a variety of these diseases can coexist, partly mimicking each other while contributing to and distorting symptomatic expressions. To understand the processes involved, it is necessary to unravel signalling pathways, complex interaction networks and metabolic alterations involving a plethora of anatomical components. When addressing such largely obscure mechanisms, primary data mainly based on genomics and differential gene expression patterns turns out to be of limited usefulness. Numerous direct as well as very indirect processes modulate and dissociate gene expression from protein functions and physiological effects. Proteomics approaches that utilise metabolic labelling and high-throughput mass spectrometry to provide proteome dynamics data need to be utilised. However, the data thus gathered encompasses a complex assembly of numerous types of intermixed cells, representing biological processes that occur in both time and space across several scalar levels. The complexities represented are such that to analytically approach these diseases, a systems standpoint becomes necessary. This implies multiple experimental interrogations in an iterative interplay between experimentation and modelling. While this may be reasonably considered in the context of in vitro systems, it can hardly be contemplated when addressing CNS tissues from heterogeneous human origins, thereby imposing serious constraints upon the investigation of human cognitive disorders. In this article, the authors expose a paradigm that addresses and alleviates at least some of these major difficulties. Based on the reasoned utilisation of trait animal models and human material, this approach has already started to deliver novel and directly exploitable knowledge.

\section{Introduction}

\section{$\nabla$}

Schizophrenia (SZ) and major depressive disorder (MDD) are severe neuropsychiatric diseases affecting approximately $10 \%$ of the world population. Family, twin, and adoption studies strongly support the presence of inheritable components $[4,72]$ in interaction with environmental factors $[49,66]$ in their aetiologies. However, the boundaries between various forms of these neuropsychiatric disorders remain unresolved, all the more so since they share major co-morbidites with each other as well as with other mental disorders [20].

Numerous genetic studies, including linkage scans and their meta-analyses, candidate gene association analyses, gene expression and genome-wide association studies (GWAS), have identified specific genes/markers and chromo- somal regions for these diseases [57,64,82]. However, the numerous, and often difficult to confirm, associations with rare copy-number variants affecting many genes, each of which only contributes a small risk, reveal the highly polygenic aetiology of these diseases [25].

Evidence from in vivo imaging and electrophysiologic studies, and from examination of post mortem tissue, indicate that SZ is characterized by selective impairments of the synaptic machinery within cerebral cortical circuits. Though many brain regions may be affected, 2 regions in which there is strong convergence across these levels of inquiry are the dorsolateral prefrontal cortex (DLPFC) and primary auditory cortex (AI) [73].

Mood disorders on the other hand are characterized by specific glial pathologies. Histopathological post mortem findings consistently show 
reductions in glial cell density or glial cell numbers in prefrontal brain regions, such as the (subgenual) anterior cingulate cortex, the orbitofrontal cortex, and the DLPFC in association with reduced prefrontal gray matter. Furthermore, specific astrocytes and oligodendrocytes histopathological alterations, such as marked reductions in amygdala's oligodendrocytes densities in MDD, and microglial alterations in bipolar disorder (BD), including manic episodes, have also been consistently reported [68].

\section{Can semiology reflect functional reality?}

Synaptic plasticity, the regulation of neuronal excitability, neurovascular coupling and the homeostasis of networks dynamics (noise-induced propagation, signal pruning, synchronization, etc.). $[33,44]$ involve the active participation of astrocyte populations $[3,18]$. Neuromodulation is a fundamental process that regulates synaptic transmission, neuronal networks activities and behaviour and it begins to appear that slow-signalling glia modulate fast synaptic transmission and neuronal firing to impact behavioural outputs, including neurological and psychiatric conditions [29]. Indeed, the adult brain rapidly and reversibly adapts its synaptic architecture to functional needs [24] and astrocytes are involved in these dynamic processes [7] as well as in the aetiology of several neurological disorders, including SZ $[28,54]$, MDD [50] and mood disorders [41], among other dysfunctions [12].

Hence, from an investigative standpoint, complex multifactorial neuropsychiatric diseases such as SZ and MDD cannot be reduced to either predominantly synaptic or predominantly glial defects since, in both cases, the interplays between non-neuronal and neuronal components are likely to be dynamically impacted and retroact on each other ( $\bullet$ Fig. 1) $[16,70]$ both in time and in space (cerebral anatomy) across several scalar levels (from metabolic to structural aspects) $[47,67,76]$. Given this reality, it appears necessary to analytically approach these diseases from a systems standpoint.

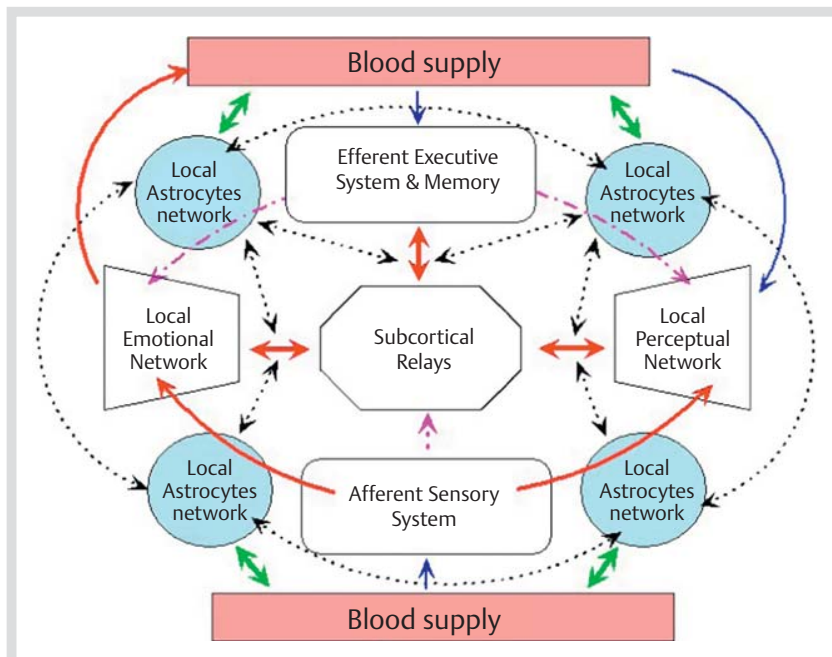

Fig. 1 Schematic overview of the interplay and cross-talk patterns that characterise the "cognitive system". Clear boxes represent function-associated neuronal networks. Heavy red arrows indicate direct inputs and feedbacks (double headed arrows) while magenta dotted arrows indicate modulating inputs between neuronal networks. The dotted double-headed black arrows indicate the reflective long range and short-range modulation roles played by local astrocyte networks upon neuronal and glial networks activities, synchronization, as well as upon vascular flow, ionic and nutrients fluxes (blue arrows) and blood-brain barrier homeostasis.
The rapid accumulation of considerable amounts of detailed biological information makes it increasingly possible to investigate the biological networks/pathways associated with complex diseases at a systems level. Furthermore, recent studies reveal striking correlations between the functional attributes of gene products or components networks and the features of the diseases they associate with [78]. These correlations are more extensive and stronger than previously thought [72] and there is a rising appreciation of the modular network organization of proteins underlying traits or mutational phenotypes, and how to exploit such protein modularity. But this requires the production of biologically relevant dynamics data feeding into biological modelling approaches capable of predicting molecular and physiological consequences that can then be experimentally verified.

\section{The Necessity for Quantitative Proteome Dynamics as Primary Data}

$\nabla$

Some understanding of the alterations affecting the components that do the actual biological work becomes crucial in any attempts to unravel the mechanisms that might be associated with a complex and highly heterogeneous phenotype. Indeed, when addressing largely obscure biological mechanisms, such as those associated with SZ or MDD, primary data mainly based on differential gene expression patterns turns out to be of limited usefulness. Numerous direct as well as very indirect processes modulate and dissociate gene expression from protein functions and actual physiological effects.

\section{Epigenetic modulation}

Short and medium term epigenetic changes carried out by specific proteins and interpreted by additional proteins do ultimately affect the expression of individual genes. One example of such proteins is the methyl-CpG-binding protein 2 (MeCP2), a transcriptional repressor that inhibits gene expression through DNA methylation and histone acetylation, that has also been shown to interact with RNA to influence alternative splicing [83]. Current research suggests that homeostatic regulation of MeCP2 is critical for the maintenance of CNS functions (neuronal maturation, dendritic morphology, and synaptic transmission) and MeCP2 mis-regulation results in abnormal behavioural and neurophysiological phenotypes [56].

\section{Transcriptional \& translational interference}

Concurrently, the discoveries on non-coding RNA (ncRNA) have changed the landscape of human genetics and molecular biology. Over the past 10 years it has become clear that several classes of ncRNAs, such as small interfering RNAs (siRNAs), microRNAs (miRNAs), PIWI-associated RNAs, small nucleolar RNAs (snRNAs) and transcribed ultra-conserved regions, are implicated in many physiological cellular processes, acting as antisense trans-acting regulators of gene expression (for example, site-specific RNA modifications and RNA-mediated silencing of gene transcription/RNA translation), and contribute to molecular alterations in pathological conditions, including cognitive diseases $[10,74]$. One such example is miR-206, a miRNA that targets tubulin polymerization-promoting protein (TPPP/p25), essential for the differentiation and maintenance of oligodendrocytes [42]. 


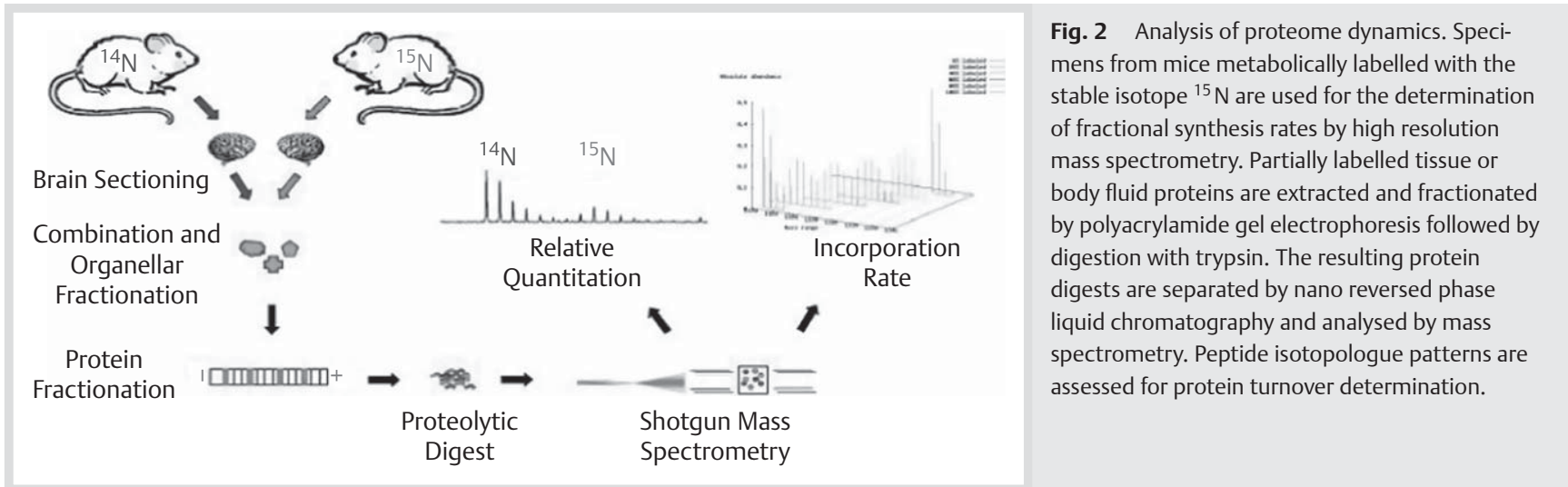

\section{Pleiotropic multi-protein complexes modulate local CNS architecture \& functions}

In addition, the cellular localisation, functional activities as well as the turnover rates of many key regulatory proteins are rapidly and tightly modulated through processes, such as polarised delivery, phosphorylation and ubiquitination, entirely independent from gene expression. The connexins, which form intercellular gap-junctional communications, are a prime example. Neuroglial cells are central elements for the brain microarchitecture and CNS functional activities are regulated through anatomical domains formed by astroglial membranes covering synapses and establishing contacts with neuronal membranes as well as with blood vessels through connexin channels and gap-junctions [75]. Astrocytes shape the grey matter by dividing it into relatively independent structural units. Protoplasmic astrocytes occupy their own territory and create micro-anatomical domains within the limits of their processes. The astroglial domains are further integrated into astroglial syncytia through gap junctions localised on the peripheral processes where astrocytic domains overlap. Thus, connexin channels form pathways for intercellular diffusion of many molecules, instrumental for long-range signalling, further contributing to the hierarchical organisation of the brain, some aspects of which are uniquely hominid [60]. Neurons, glial cells and endothelial cells express multiple connexins. Because an individual gap junction channel is composed of 12 connexins ( 6 in each adjoining cell), cells that express multiple connexins can produce mixed channels that have unique permeability and gating characteristics, provided the connexins are able to hetero-oligomerise. However, the rules that govern connexin oligomerisation and compatibility are complex and largely regulated by cellular mechanisms that restrict the formation of heteromers by otherwise compatible connexins [39].

\section{Bridging the gap through proteome metabolic labelling} All this considerably broadens the already wide gap that separates gene expression patterns from actual physiological mechanisms. Hence, what becomes important when addressing poorly defined pathological mechanisms is not so much which genes are expressed, when and at what level. It is much more a matter of which proteins, in what state, in association with which interaction partners, in the presence of which other potential partners and with what turnover rates. This requires quantitative proteome analysis of defined brain structures [48] using a shotgun-mass spectrometry (shotgun-MS) approach combined with stable isotope metabolic labelling $[46,47]$ for the accurate quan- titative comparative analysis of protein expression and turnover rates (0 Fig. 2).

Besides providing a direct entry into dysfunctional CNS pathways $[2,31]$, such proteome dynamics data can also yield biomarkers [49] allowing better diagnosis and patient stratification as well as improved treatments for disorders such as SZ and MDD, the diagnosis of which remains largely based on semiology (clinical observations as opposed to pathological examination), leading to late detection, poor prediction and trial and error treatments.

However, in cases where disease-related proteomics alterations can be found within a relatively specific brain structure, such as the DLPFC, the use of classical proteome dynamics approaches restricts the ability to determine whether the cellular distribution of other proteins which may contribute to, or compensate for, cognitive dysfunctions are concurrently altered within a given compartment. Hence, more effectively mapping the pathology within the cerebral regions showing protein alterations in subjects with SZ or MDD would clearly benefit from an approach with the ability to detect small structures with high spatial resolution, with biochemical selectivity, and with the ability to assess the relative expression levels of multiple proteins within the identified structures. This can be provided by the utilisation of confocal microscopy, which has markedly enhanced the simultaneous visualization of multiple markers of CNS fine structures by fluorescent immunohistochemistry in tissue sections [73].

\section{The Modelling Approaches Required}

\section{$\nabla$}

Disease-related proteomics alterations in post mortem cerebral tissues and/or cerebrospinal fluid from affected subjects more often than not reveal the consequences of an established pathology rather than its causes [38]. Furthermore, cerebral tissue samples, whether obtained from deceased patients or from animal models, are a complex assembly of different types of neuronal, glial and microgial cells intermixed with endothelial cells and pericytes [32]. Hence, whatever the nature of the proteomics alterations convincingly detected, these must now be associated with their most-likely cellular contexts to then have a chance to reverse engineer the events that could have given rise to the observed alterations and predict their most plausible functional consequences. This requires the utilisation of modelling approaches collectively known as "Systems Biology". 
In just over a decade, systems biology has moved from being a disparate set of ideas to a key feature of research and funding priorities [37]. Systems biology explores how parts of biological entities function and interact to give rise to the behaviour of the system as a whole. But there are no inherent limits to the levels at which "a system" may be defined. In fact, there is no such thing as "a system" because structures that are parts of one system (a transport vesicle in a cell) may form systems in their own right at a different level of integration (in the contexts of receptor trafficking and targeted surface expression). In addition to addressing the relationship between structure and function from the nano- to the macro-scale, systems biology interprets biological phenomena as dynamic processes the mechanisms and consequences of which depend on the behaviour of the living entity studied. This ranges from sub-microseconds for molecular-level interactions to days, months, and even years for the development of a disease in humans. The complexities represented are such that descriptive intuition often fails and the combinatorial problems to be addressed become serious challenges [55].

\section{The distinctions between mathematical and heuristic modelling approaches}

2 broad approaches to systems biology currently exist: the frequently followed mathematical procedures [43] and the more rarely encountered heuristic approaches [21-23], both of which are largely regarded as mutually incompatible.

Indeed, "heuristics" is a problem solving approach evaluating each step in a process, searching for satisfactory solutions rather than for optimal solutions, using all available qualitative information. Thus, heuristic modelling starts from accumulated knowledge to produce a model capable of describing the biological events and the mechanisms that generated the observed experimental data and predict their modifications associated with a different outcome.

In contrast, mathematical modelling starts from quantitative data to produce models capable of reiterating this data and predict the outcome of a different experimental paradigm. However, while their outputs are clearly different, the 2 modelling approaches, far from being incompatible can actually be complementary.

Nevertheless, models are aids to thought, not a replacement for it. Irrespective of the modelling approach utilised, the major difficulty presented by complex biological systems is that, on the basis of our present level of understanding, models remain very partial and can only be viewed as approximations of biological reality. The more complex this reality, the coarser the models will be. Thus, the predictions of a model must imperatively be experimentally tested against the biological reality it is deemed to represent.

\section{The intrinsic limitations of biological modelling approaches}

Mathematical modelling must be used with care. The fact that a model reproduces an observed behaviour does not mean that the mechanisms inferred are major contributors or are even involved at all. All that such models can do is illustrate quantitative plausibility. This takes a particular importance in situations where the structural characteristics and the nature of the mechanisms potentially involved are largely unknown, as is the case for the pathogenesis and clinical progression of most cognitive disorders.
Mathematical approaches are parametric by nature. This has 2 major consequences. First, acquiring information sufficiently detailed so as to allow definition of 1 ) the relevant quantitative parameters and 2) the driving inputs associated with complex reaction networks requires multiple experimental interrogations of the system studied as well the gathering of time-series data [9]. While this may be reasonably considered for relatively homogenous in vitro cellular systems (cell-lines), it can hardly be contemplated for complex multi-cellular in vivo systems. Secondly, while remarkably efficient for establishing interactions rules and dynamics between components, elucidating and quantifying robustness, adaptation, control and evolvability within identified networks [51], mathematical approaches perform poorly in the discovery of the structural characteristics and the nature of complex physiological mechanisms associated with illdefined multi-cellular interactions [8].

Heuristic modelling on the other hand can be very effectively used to perform this reverse engineering task [63]. However, this modelling approach should also be used with extreme caution. Being non-parametric, it functions through the systematic elimination of all potential mechanisms that cannot adequately explain the experimental data. Only those that do so while resisting invalidation by currently available information/ knowledge are retained and further utilised. However, the fact that a model adequately explains the biological origins of experimental data while resisting refutation does not mean that the mechanisms described could be correct or even have the least reality. All that such models can do is to propose plausible, unrefuted theoretical explanations. However, heuristic models often make detailed molecular and physiological predictions (what should now be observed, where, when how and why) that can then be directly experimentally verified [34].

Heuristic models suffer from a further major defect. They cannot allow to directly assess, let alone to quantify, the dynamics, robustness, adaptability, and fine controls of the biologically validated mechanisms they describe. Nevertheless, the mechanisms and associated events having been partly elucidated and biologically verified, they are now open to further analysis through mathematical modelling.

Hence, in our approach, heuristic modelling plays the role of an architect (defines the nature, the structure, the functionalities and the contextual constraints of the construct) while mathematical modelling plays the role of an engineer (reveals the dynamics and robustness of the structure while defining the set of parameters sufficient to give rise to similar or very different phenotypes).

\section{The Preferred Experimental Approach} $\nabla$

The exploration of higher levels of physiological functions through exploitation of experimental data using systems biology necessarily implies an iterative interplay between experimentation and modelling. As indicated above, irrespective of the approach followed, modelling will necessarily lead to further intermediate experimental data gathering, be it for parametric requirements or for biological verifications.

While this does not present major difficulties in the context of in vitro systems, it imposes serious constraints upon the investigation of human cognitive disorders. Not only is the necessary experimental material relatively scarce, it can seldom be obtained at the clinical stages and with the phenotypic charac- 
Table 1 The major differences that distinguish human and rodent neocortical organisation in terms of functional anatomy (constructed from 14, 71 and 60). Although unlikely to negatively impact the possibility of using rodent-derived experimental data to infer plausible pathophysiological mechanisms attached to human disorders, these differences and their likely consequences must imperatively be taken into consideration during modelling tasks under penalty of generating highly misleading constructs. WM: neocortical white matter.

\begin{tabular}{lll}
\hline CNS structural components & Humans (adults) & Rodents (mature) \\
\hline WM interstitial neurons & high densities & poorly developed \\
\hline WM parvalbumin interneurons & absent & very prominent \\
\hline MW calretinin interneurons & most prominent & median representation \\
\hline neocortical astrocytes diversity & 4 GFAP + subclasses & 2 GFAP + subclasses \\
\hline protoplasmic astrocytes & Large $\&$ complex & 16 -fold smaller; 10 -fold fewer processes \\
\hline Ca ${ }^{2+}$ waves propagation & $43.4 \pm 4.7 \mu \mathrm{m} / \mathrm{s}$ & 5 -fold slower \\
\hline astrocytic domain size & $270 \times 10^{3}$ to $2 \times 10^{6}$ synapses & $20 \times 10^{3}-120 \times 10^{3}$ synapses
\end{tabular}

teristics required [58]. Furthermore, while fraught with a multiplicity of confounding factors, such as alcohol and drug abuses or undefined effects of environmental characteristics, the majority of post mortem study subjects will have been medicated at some stage of their illness, making it particularly difficult to coherently approach the pathophysiological mechanisms. Hence, the recourse to trait animal models appears unavoidable. All the more so since, in the CNS, the complex regulation mechanisms of gene expression, protein interactions and their modulation by environmental and external factors remain largely obscure. But the limits of such models must be carefully considered and incorporated into the overall experimental strategy. In effect, while trait animal models are necessary to allow the experimental investigations and systems biology analyses associated with the modelling tasks to be performed, they should not be retained beyond this initial modelling phase. All further adaptations of the systems models should be carried out using human tissue and clinical data. There are at least 2 reasons for this.

\section{Anatomic and functional considerations}

There is converging evidence that a number of psychiatric disorders, including SZ and MDD, are associated with white matter (WM) abnormalities. However, there is a considerable heterogeneity of results, both within and between existing studies [79]. The WM underlying the cerebral neocortex is highly developed in the human brain and occupies a much larger volume than in other mammals [14]. Although the dominant components of the WM are the complex fibre tracts, the ensheathing myelin and supporting glia, there are also large numbers of dispersed neurons, the "interstitial neurons". The conflicting reports on the changes of interstitial neurons density in SZ were reviewed by Eastwood and Harrison [15], who observed a density increase in the superficial WM and no change in deeper compartments. The major differences that functionally distinguish human from rodent neocortical organisation are summarised in 0 Table 1. Interstitial neurons are prominent in primate WM but poorly developed in rodents and this may reflect a direct correlation between the size of the cortical grey matter, the amount of WM connecting the neocortex, and the number of interstitial neurons. Of the 3 calcium-binding proteins present in the cortical grey matter, calbindin $(\mathrm{CB})$, calretinin $(\mathrm{CR})$ and parvalbumin (PV)], only CB and CR are expressed in interstitial neurons within the superficial and deep adult human WM. Here, CR+ interstitial neurons are the most prominent cell population while PV is not found in the adult human WM [71]. In addition, it is now recognized that human cortical astrocytes are structurally and functionally more complex and more diverse than those of rodents [60]. The volume of astrocytic domains in rodents has been reported to range from $14700 \mu \mathrm{m}^{3}$ to $22906 \mu \mathrm{m}^{3}$ in the cortex and $65900 \mu \mathrm{m}^{3}$ to $85300 \mu \mathrm{m}^{3}$ in the hippocampus $[11,30]$. However, while organization into non-overlapping domains is similar in humans and rodents and synaptic density is relatively preserved, with approximately $1397 \times 10^{6}$ synapses $/ \mathrm{mm}^{3}$ in rodents and $1100 \times 10^{6}$ synapses $/ \mathrm{mm}^{3}$ in the cortex of man [13], human astrocytes can cover approximately 5-fold more synapses within a single domain than rodent astrocytes [60]. If an astrocytic domain is considered as an elementary brain unit that monitors, integrates, and potentially modifies the activity of a contiguous set of synapses, this glio-neuronal unit in human contains far greater numbers of synapses, and is thus capable of carrying out more complex processing per glio-neuronal unit, than that of rodents.

These unique functional features of human neocortical architecture point out the limitations of using rodent models to study human cognitive disorders. While unlikely to negatively impact the possibility of using rodent-derived experimental data to infer plausible pathophysiological mechanisms associated with the pathogenesis and progression of the human disorders, these unique features will most probably deeply affect the dynamics, the modes of control and the anatomical specificities of these mechanisms and, ultimately, the associated forms of clinical development. Hence, beyond the initial modelling phases, the continued recourse to trait animal models is likely to become highly misleading if not deleterious.

\section{Pathophysiological relevance of trait animal models}

Despite considerable efforts, there is a lack of animal models for psychiatric disorders, with only a few well-established, in particular with respect to MDD \& chronic stress.

For SZ, it is difficult to elicit in animal models a spectrum of symptoms that could reflect the human condition, and in particular auditory and/or visual hallucinations concurrently with impaired multisensory integration [80]. This is one of the main reasons why researchers have focused mainly on the analysis of human post mortem brain tissues and body fluids from SZ patients. However, SZ is a disorder that progresses transiently in brief stages and afterwards comes to a standstill. Explanations why clinical expression is delayed until adult life, or at least adolescence, remain speculative. A considerable body of evidence from neuropathological, imaging and genetic studies indicate that SZ pathology exists at a molecular, cellular and neuronal circuit level [27]. But a plethora of brain abnormalities have been described in association with SZ and findings in first-episode patients are less robust than in chronic patients [61]. In addition, since concordance rate in monozygotic twins amounts to about $50 \%$, some as yet poorly defined environmental risk factors 
(neurotrauma, drugs/alcohol abuse, psychotrauma, etc.) appear to be a necessary "second hit" to trigger/drive the disease process in a genetically predisposed individual [1]. In humans, non-competitive N-methyl-D-aspartate (NMDA) receptor antagonists, such as phencyclidine (PCP) or ketamine, reproduce the schizophrenia-like psychosis in healthy volunteers, including positive symptoms, negative symptoms and cognitive dysfunction [17] and exaggerate the psychosis in SZ patients [59]. Many mouse models have been developed through either genetic manipulations $[35,65]$ or pharamacological interventions $[5,62]$, presenting various aspects of SZ-like phenotypes, both in terms of behaviour and brain anatomy defects. But most are considered to be of little translational relevance [81].

The problem may reside much more with our vision of the disorder rather than with the actual animal models. In human populations, the risk of developing SZ is clearly enhanced following prenatal and/or perinatal exposure to various environmental insults, including maternal exposure to stress, infection and/or immune activation, nutritional deficiencies and obstetric complications [52] within a genetic background involving numerous genes, each contributing a small risk. Hence, it is highly unlikely for a single animal model to satisfy all the necessary clinical requirements and it is probably an error to expect any animal model to do so. All the more so since, in complete opposition to human population, any rodent animal model that is inbred presents a homogeneous phenotype and spends its life in a controlled environment.

Animal models are, and can only be considered as approximations of phenomena of interest that are more tractable for experimental investigation. Thus, to be useful, animal models of any given psychiatric disorder need not fully recapitulate the disease. Indeed, given the human uniqueness of these disorders, that would actually be impossible. Hence, in mouse models of SZ, 2 main factors must be considered: the genetic and clinical data upon which the model is based, and the methods for characterizing the model.

It remains likely that the brain structural abnormalities observed in SZ have several origins. Besides neurodevelopmental anomalies, alterations in cortical plasticity and maturation processes may also occur over the course of the disease [26]. It would thus appear reasonable to utilise animal models that successfully capture different but overlapping spectra of structural and functional brain abnormalities implicated in SZ, some of which can be normalized by acute and/or chronic drug treatments. Here, the wealth of epidemiological data regarding the aetiology, neurobiology and psychopharmacology of SZ as well as other cognitive pathologies can be of considerable utility.

However, while these animal models can provide indispensable experimental avenues for the elucidation of neurobiological mechanisms likely to be involved in the pathogenesis and progression of neuropsychiatric disorders, each only partly recapitulates defined semiological aspects of the human disease. Therefore, their utilisation should not extend beyond the initial heuristic modelling and validation phases.

\section{Perspectives}

$\nabla$

Currently the diagnostic and follow-up of most neuropsychiatric disorders is based on the identification of cluster of symptoms and scales. Psychiatric conditions, such as SZ, MDD, mood and anxiety disorders, are characterised by symptoms that arise involuntarily as opposed to behavioural disorders (substance abuse, anorexia nervosa, etc.) in which choices are essential. Yet, no systematic relationship appears to exist between the diseaselike character of a psychiatric disorder and its heritability while many behavioural disorders seem to be more heritable than conditions commonly construed as diseases [6]. This suggests that, among psychiatric disorders, there is no close relationship between the strength of genetic influences and the aetiologic importance of volitional processes. Furthermore, the genetic variants implicated so far account for only a fraction of disease liability, a phenomenon not limited to psychiatric phenotypes but characteristic of all complex genetic traits studied to date [69]. For many of these conditions, it remains impossible to identify individuals at risk or to easily make an accurate diagnosis. This is in part due to the fact that the aetiopathogenesis of many illnesses affecting the CNS remains unclear and in part to the fact that a variety of these diseases can coexist and partly mimic each other, each capable of contributing to and distorting symptomatic expression. CNS disorders are likely to arise from the dynamic dysregulation of several regulatory networks, leading to physiologic and metabolic alterations, reflecting complex perturbations of the "system". Thus, it appears that genomic and gene expression approaches will not suffice to unravel the basis or characterise the pathogenesis of complex neuropsychiatric illnesses. To understand these physiological processes, it is necessary to unravel signal transduction pathways, complex interaction networks and metabolic alterations on the level of the proteins that carry out the actual physiological tasks in order to then coherently access the much more intractable levels of genomic DNA, subject to cryptic mutational and epigenetic effects, and mRNA, subject to intricate regulatory and splicing mechanisms. In this context, proteomics approaches that utilise metabolic labelling and high-throughput mass spectrometric protein identification can reveal quantitative and qualitative protein expression levels, posttranslational modifications and protein-protein interactions patterns together with their dynamics, allowing the elucidation of disease-associated molecular mechanisms [31]. However, it is CNS tissue samples that must be analysed and the proteome complexity represented is such [19] that systems biology approaches, that make extensive use of modelling in order to represent and understand complex interactions of biological entities, will be analytically inescapable.

Yet, it is amply clear that, since both the structure and the nature of the event-associated mechanisms potentially involved remain largely obscure, mathematical modelling cannot be fruitfully used as a first-line approach. It becomes necessary to first implement heuristic modelling which, using disease-related proteomics alterations and confocal microscopy imaging data that distinguish control from affected tissues, will provide the means to identify, characterize and subsequently validate the broad structure and the nature of the event-associated mechanisms that need to be explored in detail [34]. Once this is achieved, mathematical modelling can then be fruitfully and efficiently utilised, making full use of the dynamic components of the data to help reveal the interactions rules between components, elucidating and quantifying robustness, adaptation, and broad controls within the biologically validated mechanisms. This implies the ability to repeatedly access equivalent tissue samples subsequently to more or less altered experimental paradigms, thus precluding the utilization of human tissue and imposing the recourse to experimental animal models. 


\section{Conclusions}

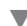

Over the last 2 decades, the progresses made with respect to the aetiopathogenesis of psychiatric disorders have been highly influential in shaping animal models for these diseases $[45,77]$. The use of selective lesions in adult animals and the acute or chronic administration of psychotomimetic agents have proven to be inaccurate, if not inadequate, in capturing the aetiological mechanisms or the ontology needed for an appropriate understanding of the disease [53]. This has motivated the establishment of animal models allowing to address hypotheses concerning the subtle histopathological and neuroanatomical findings revealed in human post mortem and imaging studies, as well as the contribution of genetic and environmental risk factors [52]. The spectrum of identified structural and functional brain abnormalities encountered in these animal models, and the fact that some of them show a developmental delay in phenotype emergence $[36,40]$ leads to the possibility of investigating particular aspects of defined psychiatric conditions. Hence, provided metabolic labelling is utilized, investigations associating proteomics and systems biology can be carried out with sufficient depth to arrive at a functional understanding of the pathomechanisms involved and, most importantly, reveal the identity, the state and the dynamics of their proteomic markers. However, while these animal models may be instrumental in shedding light upon critical dysfunctional processes, investigation of the human brain remains essential to determine the relevance of the identified processes to the human pathology, as assessed through the absence/presence and the state of the corresponding proteomic markers. If appropriate, the relevant animal model can be further utilized to carry out heuristic and mathematical modelling of the probable modes of control regulating common major processes, thus providing some understanding of the causes behind the symptomatology of these disorders, and, eventually, help to establish early preventive interventions that could successfully reduce the risk of developing these disabling brain disorders in exposed individuals.

\section{Conflict of Interests}

\section{$\nabla$}

The authors declare no conflicts of interest.

\section{References}

1 Arguello PA, Markx S, Gogos JA et al. Development of animal models for schizophrenia. Dis Model Mech 2010; 3: 22-26

2 Beasley CL, Pennington K, Behan A et al. Proteomic analysis of the anterior cingulate cortex in the major psychiatric disorders: Evidence for disease-associated changes. Proteomics 2006; 6: 3414-3425

3 Ben Achour S, Pascual O. Glia: The many ways to modulate synaptic plasticity. Neurochem Int 2010

4 Bergemann $E R$, Boles RG. Maternal inheritance in recurrent early-onset depression. Psychiatr Genet 2010; 20: 31-34

5 Berretta S, Benes FM. A rat model for neural circuitry abnormalities in schizophrenia. Nat Protoc 2006; 1: 833-839

6 Bienvenu OJ, Davydow DS, Kendler KS. Psychiatric 'diseases' versus behavioral disorders and degree of genetic influence. Psychol Med 2010; $1-8$

7 Bosler O, Girardet C, Sage-Ciocca D et al. [Mechanisms of structural plasticity associated with photic synchronization of the circadian clock within the suprachiasmatic nucleus]. J Soc Biol 2009; 203: 49-63

8 Bruggeman FJ. Systems biology: from possible to plausible to actual models. FEBS J 2009; 276: 885
9 Cedersund G, Roll J. Systems biology: model based evaluation and comparison of potential explanations for given biological data. FEBS J 2009; 276: 903-922

10 Choudhuri S. Small noncoding RNAs: biogenesis, function, and emerging significance in toxicology. J Biochem Mol Toxicol 2010; 24: 195-216

11 Chvatal A, Anderova M, Kirchhoff F. Three-dimensional confocal morphometry - a new approach for studying dynamic changes in cell morphology in brain slices. J Anat 2007; 210: 671-683

12 De Keyser J, Mostert JP, Koch MW. Dysfunctional astrocytes as key players in the pathogenesis of central nervous system disorders. J Neurol Sci 2008; 267: 3-16

13 DeFelipe J, Alonso-Nanclares L, Arellano JI. Microstructure of the neocortex: comparative aspects. J Neurocytol 2002; 31: 299-316

14 Defelipe J, Fields RD, Hof PR et al. Cortical white matter: beyond the pale remarks, main conclusions and discussion. Front Neuroanat 2010; 4: 4

15 Eastwood SL, Harrison PJ. Interstitial white matter neuron density in the dorsolateral prefrontal cortex and parahippocampal gyrus in schizophrenia. Schizophr Res 2005; 79: 181-188

16 England PM. Bridging the gaps between synapses, circuits, and behavior. Chem Biol 2010; 17: 607-615

17 Enomoto T, Noda $Y$, Nabeshima T. Phencyclidine and genetic animal models of schizophrenia developed in relation to the glutamate hypothesis. Methods Find Exp Clin Pharmacol 2007; 29: 291-301

18 Fellin T. Communication between neurons and astrocytes: relevance to the modulation of synaptic and network activity. J Neurochem 2009; 108: 533-544

19 Filiou MD, Bisle B, Reckow $S$ et al. Profiling of mouse synaptosome proteome and phosphoproteome by IEF. Electrophoresis 2010; 31: 1294-1301

20 Fineberg NA, Saxena S, Zohar J et al. Obsessive-compulsive disorder: boundary issues. CNS Spectr 2007; 12: 359-364, 367-375

21 Gadal F, Bozic C, Pillot-Brochet $C$ et al. Integrated transcriptome analysis of the cellular mechanisms associated with Ha-ras-dependent malignant transformation of the human breast epithelial MCF7 cell line. Nucleic Acids Res 2003; 31: 5789-5804

22 Gadal F, Starzec A, Bozic C et al. Integrative analysis of gene expression patterns predicts specific modulations of defined cell functions by estrogen and tamoxifen in MCF7 breast cancer cells. J Mol Endocrinol 2005; 34: 61-75

23 Geier F, Timmer J, Fleck C. Reconstructing gene-regulatory networks from time series, knock-out data, and prior knowledge. BMC Syst Biol 2007; $1: 11$

24 Girardet C, Blanchard MP, Ferracci G et al. Daily changes in synaptic innervation of VIP neurons in the rat suprachiasmatic nucleus: contribution of glutamatergic afferents. Eur J Neurosci 2010; 31: 359-370

25 Glessner JT, Hakonarson H. Common variants in polygenic schizophrenia. Genome Biol 2009; 10: 236

26 Gourion D, Gourevitch $R$, Leprovost JB et al. [Neurodevelopmental hypothesis in schizophrenia]. Encephale 2004; 30: 109-118

27 Gross G, Huber G. Schizophrenia: neurodevelopmental disorder or degenerative brain process? Fortschr Neurol Psychiatr 2008; 76 (Suppl 1): S57-S62

28 Halassa MM, Fellin T, Haydon PG. The tripartite synapse: roles for gliotransmission in health and disease. Trends Mol Med 2007; 13: 54-63

29 Halassa MM, Fellin T, Haydon PG. Tripartite synapses: roles for astrocytic purines in the control of synaptic physiology and behavior. Neuropharmacology 2009; 57: 343-346

30 Halassa MM, Fellin T, Takano $\mathrm{H}$ et al. Synaptic islands defined by the territory of a single astrocyte. J Neurosci 2007; 27: 6473-6477

31 Hambsch B, Chen BG, Brenndorfer J et al. Methylglyoxal-mediated anxiolysis involves increased protein modification and elevated expression of glyoxalase 1 in the brain. J Neurochem 2010; 113: 1240-1251

32 Haseloff RF, Blasig IE, Bauer HC et al. In search of the astrocytic factor(s) modulating blood-brain barrier functions in brain capillary endothelial cells in vitro. Cell Mol Neurobiol 2005; 25: 25-39

33 Hauber $W$. Dopamine release in the prefrontal cortex and striatum: temporal and behavioural aspects. Pharmacopsychiatry 2010; 43 (Suppl 1): S32-S41

34 Iris F, Gea M, Lampe PH et al. [Production and implementation of predictive biological models]. Med Sci (Paris) 2009; 25: 608-616

$35 \mathrm{Karl} T$, Chesworth R, Duffy L et al. Acoustic startle response and sensorimotor gating in a genetic mouse model for the Y1 receptor. Neuropeptides 2010; 44: 233-239 
36 Koenig JI, Elmer GI, Shepard PD et al. Prenatal exposure to a repeated variable stress paradigm elicits behavioral and neuroendocrinological changes in the adult offspring: potential relevance to schizophrenia. Behav Brain Res 2005; 156: 251-261

37 Kohl P, Crampin EJ, Quinn TA et al. Systems biology: an approach. Clin Pharmacol Ther 2010; 88: 25-33

38 Korolainen MA, Nyman TA, Aittokallio T et al. An update on clinical proteomics in Alzheimer's research. J Neurochem 2010; 112: 1386-1414

39 Koval M. Pathways and control of connexin oligomerization. Trends Cell Biol 2006; 16: 159-166

40 Lee PR, Brady DL, Shapiro RA et al. Prenatal stress generates deficits in rat social behavior: Reversal by oxytocin. Brain Res 2007; 1156: 152-167

41 Lee Y, Gaskins D, Anand A et al. Glia mechanisms in mood regulation: a novel model of mood disorders. Psychopharmacology (Berl) 2007; 191: 55-65

42 Lehotzky A, Lau P, Tokesi $N$ et al. Tubulin polymerization-promoting protein (TPPP/p25) is critical for oligodendrocyte differentiation. Glia 2010; 58: 157-168

43 Liebal UW, Millat T, de Jong IG et al. How mathematical modelling elucidates signalling in B. subtilis. Mol Microbiol 2010

44 Liljenstrom $H$. Network effects of synaptic modifications. Pharmacopsychiatry 2010; 43 (Suppl 1): S67-S81

45 Lipska BK. Using animal models to test a neurodevelopmental hypothesis of schizophrenia. J Psychiatry Neurosci 2004; 29: 282-286

46 Maccarrone G, Turck CW, Martins-de-Souza D. Shotgun mass spectrometry workflow combining IEF and LC-MALDI-TOF/TOF. Protein J 2010; 29: 99-102

47 Martins-De-Souza D, Dias-Neto E, Schmitt A et al. Proteome analysis of schizophrenia brain tissue. World J Biol Psychiatry 2010; 11: 110-120

48 Martins-de-Souza D, Harris LW, Guest PC et al. The role of proteomics in depression research. Eur Arch Psychiatry Clin Neurosci 2009

49 Martins-de-Souza D, Maccarrone G, Wobrock T et al. Proteome analysis of the thalamus and cerebrospinal fluid reveals glycolysis dysfunction and potential biomarkers candidates for schizophrenia. J Psychiatr Res 2010

50 McNally L, Bhagwagar Z, Hannestad J. Inflammation, glutamate, and glia in depression: a literature review. CNS Spectr 2008; 13: 501-510

51 Menolascina F, Bellomo D, Maiwald T et al. Developing optimal input design strategies in cancer systems biology with applications to microfluidic device engineering. BMC Bioinformatics 2009; 10 (Suppl 12): S4

52 Meyer U, Feldon J. Epidemiology-driven neurodevelopmental animal models of schizophrenia. Prog Neurobiol 2010; 90: 285-326

53 Meyer U, Feldon J, Fatemi SH. In-vivo rodent models for the experimental investigation of prenatal immune activation effects in neurodevelopmental brain disorders. Neurosci Biobehav Rev 2009; 33 : 1061-1079

54 Mitterauer BJ. The syncytiopathy hypothesis of depression: downregulation of glial connexins may protract synaptic information processing and cause memory impairment. Med Hypotheses 2010; 74: 497-502

55 Moore MN, Noble D. Editorial: computational modelling of cell \& tissue processes \& function. J Mol Histol 2004; 35: 655-658

$56 \mathrm{Na}$ ES, Monteggia LM. The role of MeCP2 in CNS development and function. Horm Behav 2010

$57 \mathrm{Ng}$ MY, Levinson DF, Faraone SV et al. Meta-analysis of 32 genomewide linkage studies of schizophrenia. Mol Psychiatry 2009; 14 : 774-785

58 Niculescu $A B$. Genomic studies of mood disorders - the brain as a muscle? Genome Biol 2005; 6: 215

59 Noda Y, Mouri A, Waki Y et al. [Development of animal models for schizophrenia based on clinical evidence: expectation for psychiatrists]. Nihon Shinkei Seishin Yakurigaku Zasshi 2009; 29: 47-53

60 Oberheim NA, Takano T, Han X et al. Uniquely hominid features of adult human astrocytes. J Neurosci 2009; 29: 3276-3287

61 Peters BD, Blaas J, de Haan L. Diffusion tensor imaging in the early phase of schizophrenia: What have we learned? J Psychiatr Res 2010
62 Pietraszek M, Michaluk J, Romanska I et al. 1-Methyl-1,2,3,4-tetrahydroisoquinoline antagonizes a rise in brain dopamine metabolism, glutamate release in frontal cortex and locomotor hyperactivity produced by MK801 but not the disruptions of prepulse inhibition, and impairment of working memory in rat. Neurotox Res 2009; 16: 390-407

63 Pouillot F, Blois $H$, Iris F. Genetically engineered virulent phage banks in the detection and control of emergent pathogenic bacteria. Biosecur Bioterror 2010; 8: 155-169

64 Purcell SM, Wray NR, Stone JL et al. Common polygenic variation contributes to risk of schizophrenia and bipolar disorder. Nature 2009; 460: $748-752$

65 Radyushkin K, El-Kordi A, Boretius $S$ et al. Complexin2 null mutation requires a 'second hit' for induction of phenotypic changes relevant to schizophrenia. Genes Brain Behav 2010

66 Riley AW, Valdez CR, Barrueco $S$ et al. Development of a family-based program to reduce risk and promote resilience among families affected by maternal depression: theoretical basis and program description. Clin Child Fam Psychol Rev 2008; 11: 12-29

67 Schipke CG, Heuser I, Peters O. Antidepressants act on glial cells: SSRIs and serotonin elicit astrocyte calcium signaling in the mouse prefrontal cortex. J Psychiatr Res 2010

68 Schroeter ML, Abdul-Khaliq H, Sacher J et al. Mood disorders are glial disorders: evidence from in vivo studies. Cardiovasc Psychiatry Neurol 2010; 2010: 780645

69 Schulze TG. Genetic research into bipolar disorder: the need for a research framework that integrates sophisticated molecular biology and clinically informed phenotype characterization. Psychiatr Clin North Am 2010; 33: 67-82

70 Silver RA. Neuronal arithmetic. Nat Rev Neurosci 2010; 11: 474-489

71 Suarez-Sola ML, Gonzalez-Delgado FJ, Pueyo-Morlans $M$ et al. Neurons in the white matter of the adult human neocortex. Front Neuroanat 2009; $3: 7$

72 Sun J, Jia P, Fanous AH et al. Schizophrenia gene networks and pathways and their applications for novel candidate gene selection. PLoS One 2010; 5: e11351

73 Sweet RA, Fish KN, Lewis DA. Mapping Synaptic Pathology within Cerebral Cortical Circuits in Subjects with Schizophrenia. Front Hum Neurosci 2010; 4: 44

74 Taft RJ, Pang KC, Mercer TR et al. Non-coding RNAs: regulators of disease. J Pathol 2010; 220: 126-139

75 Verkhratsky A. Physiology of neuronal-glial networking. Neurochem Int 2010

76 Walter M, Henning A, Grimm S et al. The relationship between aberrant neuronal activation in the pregenual anterior cingulate, altered glutamatergic metabolism, and anhedonia in major depression. Arch Gen Psychiatry 2009; 66: 478-486

77 Wang L, Simpson HB, Dulawa SC. Assessing the validity of current mouse genetic models of obsessive-compulsive disorder. Behav Pharmacol 2009; 20: 119-133

78 Wang PI, Marcotte EM. It's the machine that matters: Predicting gene function and phenotype from protein networks. J Proteomics 2010

79 White T, Nelson M, Lim KO. Diffusion tensor imaging in psychiatric disorders. Top Magn Reson Imaging 2008; 19: 97-109

80 Williams LE, Light GA, Braff DL et al. Reduced multisensory integration in patients with schizophrenia on a target detection task. Neuropsychologia 2010

81 Wilson C, Terry AV Jr. Neurodevelopmental animal models of schizophrenia: role in novel drug discovery and development. Clin Schizophr Relat Psychoses 2010; 4: 124-137

82 Wray NR, Visscher PM. Narrowing the boundaries of the genetic architecture of schizophrenia. Schizophr Bull 2010; 36: 14-23

83 Young JI, Hong EP, Castle JC et al. Regulation of RNA splicing by the methylation-dependent transcriptional repressor methyl-CpG binding protein 2. Proc Natl Acad Sci USA 2005; 102: 17551-17558 